\title{
ON WRONSKIANS OF FUNCTIONS OF A REAL VARIABLE.
}

BY PROFESSOR MAXIME BôCHER.

(Read before the American Mathematical Society, August 20, 1901.)

THE important part played by the real roots of solutions of homogeneous linear differential equations of the second order is well known. When we pass to equations of higher order, it becomes necessary to consider not only the roots of the solutions of the equations, but also the roots of Wronskians* of sets of linearly independent solutions. Unless we are willing to confine ourselves to the case in which the coefficients of the differential equation, and therefore also the solutions, are analytic functions, a number of questions present themselves to us at the very outset-for instance the question whether such Wronskians can have an infinite number of roots in a given interval; and again the question to what extent the theory of the adjoint + differential equation remains valid when the coefficients of the differential equation are not assumed to be analytic but merely continuous functions. It is my object in the present paper to settle some of these questions and thus clear the way for further investigations. In doing this I have first considered the slightly more general subject of linear families of which the solutions of a homogeneous linear differential equation form a special case.

\section{\$1. Exponents.}

We will say that a function $\$ f$ of the real variable $x$ has at a point $c$ the exponent $x$ if throughout the neighborhood of $c$

$$
\text { (1) } \quad f(x)=(x-c)^{\kappa} E(x)
$$

* I shall denote the Wronskian of $m$ functions $f_{1}, f_{2}, \cdots, f_{m}$, that is the determinant whose $i$ th row is

by $W\left(f, \cdots, f_{m}\right)$.

$$
f_{1}^{[i-1]} f_{2}^{[i-1]} \cdots f_{m}^{[i-1]},
$$

$\nmid$ Adjungirte. Cf. Schlesinger, Handbuch, vol. 1, pp. 53-75, where proofs of the theorems in the case of analytic functions are given, as well as references to the original memoirs.

$\ddagger$ The functions of a real variable used in this paper need not necessarily be real. 
where $E(x)$ is continuous at $c$ and throughout its neighborhood and $E(c) \neq 0$.

I. A function cannot have two different exponents at the same point.

Throughout the present paper we will denote by $(J)$ the interval

$$
(J) \quad a \leqq x \leqq b .
$$

II. If $f(x)$ has an exponent at each point of $(J)$ it cannot have more than a finite number of discontinuities in $(J)$, nor can it have more than a finite number of roots in $(J)$.

For if $f(x)$ had an infinite number of discontinuities, or of roots, or of both, these points would have at least one limiting point $c$ in $(J)$. But since $f$ has an exponent at $c$ it is continuous and different from zero throughout the neighborhood of $c$.

We will from now on confine ourselves to the case of real exponents. If $f(x)$ has an exponent at $c$ and if $k$ is a constant not zero it is clear that $k f(x)$ has the same exponent there. If $f_{1}(x)$ and $f_{2}(x)$ have different real exponents at $c, f_{1}(x)+f_{2}(x)$ has an exponent at $c$, namely the smaller of the two exponents of $f_{1}$ and $f_{2}$. A combination of these two facts gives us the following theorem :

III. If $f_{1}(x), f_{2}(x), \cdots, f_{m}(x)$ have at $c$ real exponents no two of which are equal, and if $k_{1}, k_{2}, \cdots, k_{m}$ are constants no one of which is zero, then the function

$$
k_{1} f_{1}(x)+k_{2} f_{2}(x)+\cdots+k_{m} f_{m}(x)
$$

has an exponent at $c$, namely the smallest of the exponents of $f_{1}$, $f_{2}, \cdots, f_{m}$.

By combining II. and III. we get the further theorem

IV. If $f_{1}, f_{2}, \cdots, f_{m}$ have at $c$ real exponents no two of which are equal, then these functions are linearly independent.*

If $f(x)$ is continuous at $c$, then to say that it has the exponent zero at $c$ merely means that it does not vanish there; while instead of saying that it has the positive integral exponent $x$ at $c$ we might very well say that it has a $x$-fold root at this point. $\dagger$ The following theorem shows that the familiar test for the multiplicity of a root is valid provided the necessary derivatives exist and are continuous :

* For a special case of this theorem cf. Heffter : Lineare Differentialgleichungen, p. 236.

$\dagger$ Thus for instance the property which $\kappa$-fold roots of real analytic funtions have that on opposite sides of such a root the function has the same or opposite signs according as $\kappa$ is even or odd is at once seen to hold here if $f(x)$ is real. 
V. If throughout an interval including a point c $f(x)$ is continuous and has continuous derivatives of the first $x$ orders, a necessary and sufficient condition that $f(x)$ have the exponent $x$ at $c$ is

$$
f^{[i]}(c)=0 \quad(i=0,1, \cdots, x-1) ; \quad f^{[k]}(c) \neq 0 . *
$$

For if conditions (2) are fulfilled we have by Taylor's theorem, if we use Lagrange's formula for the remainder,

$$
f(x)=\frac{(x-c)^{\kappa}}{x !} f^{[\kappa]}[c+\theta(x-c)], \dagger
$$

where $\theta$ is a function of $x$ satisfying the inequality $0<\theta<1$. When $x \neq c, f^{[k]}[c+\theta(x-c)]$ is continuous because it is then equal to $x !(x-c)^{-\kappa} f(x)$. When $x=c$, it is also continuous since $\lim _{x=c} f^{[k]}[c+0(x-c)]=f^{[\kappa]}(c)$. Since this last quantity is not zero, (3) is a special case of (1) and therefore $f(x)$ has the exponent $x$.

Conversely, if $f(x)$ has the exponent $x$ at $c$, we see by the part of the theorem just proved that none of the quantities $f^{[i]}(c)(i<x)$ can be different from zero. That $f^{[k]}(c) \neq 0$ follows at once from $(3)$ when we let

for we then find

$$
f(x)=(x-c)^{\kappa} E(x)
$$

$$
E(c)=\frac{1}{x !} f^{[\kappa]}(c) .
$$

If we apply the theorem just proved and formula (4) not to $f(x)$ but to $f^{[j]}(x)$ we get the theorem \$

VI. If $f(x)$ has the exponent $*$ at c. and in an interval including $c$ is continuous and has continuous derivatives of the first $x$ orders, then $f^{[j]}(x)(j=1,2, \cdots, x)$ has the exponent $x-j$ at $c$, and if we write

* If $f(x)$ has an exponent, not necessarily integral, greater than $\kappa$ it can readily be proved that even though $f(x)$ has no derivatives of order higher than $\kappa$

$$
f^{[i]}(c)=0 \quad(i=0,1, \cdots, \kappa) .
$$

† This formula must be slightly modified if $f(x)$ is a complex function of the real variable $x$, the real quantity $\theta$ being then different in the real and the imaginary part of $f[k]$. No difficulty, however, is introduced hereby ; and in fact $f^{\prime}(x)$ may be complex throughout the whole of the present paper.

$\ddagger$ It should be noticed that we cannot simply say that if $f(x)$ has the exponent $\kappa$, and if $f^{\prime}(x)$ exists and is continuous, then $f^{\prime}(x)$ has the exponent $x-1$. This is seen by considering the function $x^{2}+x^{3} \sin \frac{1}{x}$ at the point $x=0$. 


$$
f(x)=(x-c)^{\kappa} E(x), f^{*}(x)=(x-c)^{\kappa-j} E_{j}(x),
$$

then

$$
E_{j}(c)=x(x-1) \cdots(x-j+1) E(c) .
$$

\$2. Linear Families and Their Bases.

Let $y_{1}, y_{2}, \cdots, y_{k}$ be functions of the real variable $x$ which in the interval

$$
a \leqq x \leqq b
$$

are linearly independent. The family of functions represented by the formula

$$
C_{1} y_{1}+C_{2} y_{2}+\cdots+C_{k} y_{k},
$$

the $C^{\prime}$ 's being arbitrary constants, we will call a linear family. By a basis of this family we understand a system of linearly independent functions $\eta_{1}, \eta_{2}, \cdots, \eta_{m}$ such that the linear family

$$
K_{1} \eta_{1}+K_{2} \eta_{2}+\cdots+K_{m} \eta_{m}
$$

is identical with (6). In particular therefore the $y$ 's form a basis. Moreover if the $\eta$ 's form a basis each of them must be a function of the family (6).

VII. A necessary and sufficient condition that the functions $\eta_{1}$, $\eta_{2}, \cdots, \eta_{m}$ belonging to the family (6) form a basis is that they are linearly independent and that $m=k$.

For expressing the $\eta$ 's as linear functions of the $y$ 's we easily see that if $m>k$ the $\eta$ 's must be linearly dependent, and therefore cannot form a basis.

On the other hand if $m<k$ and the $\eta$ 's formed a basis, the $y$ 's would form a basis of (7); and this is impossible by what we have just proved.

Finally if $m=k$, let us write

$$
\eta_{i}=\alpha_{i 1} y_{1}+\alpha_{i 2} y_{2}+\cdots+\alpha_{i k} y_{k} \quad(i=1,2, \cdots k) .
$$

Substituting this in (7), we see that every function of $(7)$ belongs to $(6)$. If the $\eta$ 's are linearly independent the determinant of the $\alpha$ 's must be different from zero. Equations (8) can therefore be solved for the $y$ 's, and these values substituted in (6). We thus see that every function of (6) belongs to (7) ; that is, (6) and (7) are identical.

Let us now impose on our linear family (6) the further restriction, that every function, except zero, of the family has a real exponent at the point $c$ of $(J)$.

Among all the functions of this family there cannot be more than $k$ different exponents for a given point $c$ of $(J)$ 
as we should otherwise, by IV., have more than $k$ linearly independent functions in the family. It is less obvious that the number of distinct exponents at $c$ cannot fall below $k$. This can, however, be proved as follows :

Suppose there were $l$ and only $l$ distinct exponents at $c$, and $l<k$. Denote these exponents in order of increasing magnitude by $x_{1}, x_{2}, \cdots, x_{l}$. Let $\eta_{1}, \eta_{2}, \cdots, \eta_{l}$ be functions which have at $c$ the exponents $x_{1}, x_{2} \cdots, x_{l}$ respectively. Since $\eta_{1}, \eta_{2}, \cdots, \eta_{l}$ do not form a basis, being less than $k$ in number, there exist functions of the family which are linearly independent of them. Let $x_{n}$ be the largest of the exponents to which corresponds a function $\bar{\eta}_{m}$ of the family linearly independent of $\eta_{1}, \eta_{2}, \cdots, \eta_{l}$, and write

$$
\eta_{m}=(x-c)^{\kappa_{m}} E(x), \quad \bar{\eta}_{m}=(x-c)^{\kappa_{m}} \bar{E}(x) .
$$

The function

may be written

$$
\eta(x)=\eta_{m}(x)-\frac{E(c)}{\bar{E}(c)} \bar{\eta}_{m}(x),
$$

$$
\eta(x)=(x-c)^{\kappa_{m}} \varphi(x),
$$

where $\varphi(c)=0$. It therefore does not have at $c$ any of the exponents $x_{1}, x_{2}, \cdots, x_{m}$, and hence, since it is a member of the family, it must have an exponent larger than $x_{m}$ and must therefore be linearly dependent on the $\eta$ 's,

$$
\eta_{m}(x)-\frac{E(c)}{\bar{E}(c)} \eta_{m}(x)=c_{1} \eta_{1}(x)+\cdots+c_{l} \eta_{l}(x) .
$$

But this makes $\bar{\eta}_{m}$ linearly dependent on $\eta_{1}, \cdots, \eta_{l}$, and this is contrary to hypothesis. We have thus proved the theorem

VIII. If in the linear family (6) every function except zero has at $c$ a real exponent, then among these exponents there exist $k$ and only $k$ distinct values.

We will speak of these $k$ quantities as the exponents of the family at $c$.

Any set of $k$ functions of the family corresponding at $c$ to these distinct exponents will form a basis. Such a basis we will call a principal basis for the point $c$.

\section{§3. Roots of Wronskians.}

Let us now assume that $y_{1}, y_{2}, \ldots, y_{k}$, besides being linearly independent throughout $(J)$, are continuous and have continuous derivatives of the first $n-1$ orders $(n \geqq k)$ throughout this interval. The same will then be true for every 
function of (6), and we will assume that no function of the family, except zero, vanishes together with its first $n-1$ derivatives at any point of $(J)$. Every function of the family will therefore, by V., have at any point of $(J)$ an integral exponent $x$, where $0 \leqq x \leqq n-1$. We will now prove a similar theorem for the Wronskian of the $y$ 's $*$

IX. At any point $c$ of $(J)$ the Wronskian

$$
W\left(y_{1}, y_{2}, \cdots, y_{k}\right)
$$

has an integral exponent $x$, where

$$
0 \leqq x \leqq k(n-k) \text {. }
$$

Let $x_{1}, x_{2}, \cdots, x_{k}$ be the exponents of the family at $c$, and let $\eta_{1}, \eta_{2} \cdots, \eta_{k}$ be a principal basis of the family at this point. Then

$$
W\left(\eta_{1}, \eta_{2} \cdots, \eta_{k}\right)=C W\left(y_{1}, y_{2}, \cdots, y_{k}\right),
$$

where $C$ is a non-vanishing constant, namely the determinant of the transformation (8). Accordingly it will be sufficient to prove that $W\left(\eta_{1}, \cdots, \eta_{k}\right)$ has an exponent $x$ at $c$ satisfying the above written inequality.

Let us write

$$
\eta_{i}(x)=A_{i}(x-c)^{\kappa_{i}}\left[1+\varphi_{i}(x)\right] \quad(i=1,2, \cdots, k),
$$

where $A_{i}$ is a non-vanishing constant and $\varphi_{i}$ a function of $x$ continuous throughout $(J)$ and vanishing at $c$. We can write further

$$
\begin{gathered}
\eta_{i}^{(j)}(x)=A_{i}(x-c)^{\kappa_{i}-j}\left[x_{i}\left(x_{i}-1\right) \cdots\left(x_{1}-j+1\right)+\varphi_{i j}(x)\right] \\
(i=1,2, \cdots, k ; j=1,2, \cdots, k-1),
\end{gathered}
$$

where the functions $\varphi_{i j}$ are continuous throughout $(J)$ and vanish at $c$. This formula follows at once from VI. when $j \leqq x_{i}$, while when $j>x_{i}$ its correctness is seen directly when we let

$$
\varphi_{i j}(x)=\frac{(x-c)^{j-\kappa_{i}}}{A_{i}} \eta_{i}^{(j)}(x),
$$

since the quantity $x_{i}\left(x_{i}-1\right) \cdots\left(x_{i}-j+1\right)$ is zero in this case.

Substituting (10) and (11) in $W\left(\eta_{1}, \cdots, \eta_{k}\right)$ we find

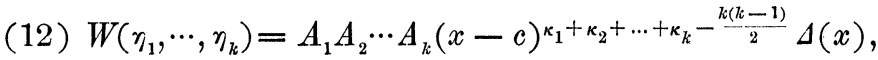
where

* It may be noted that the derivatives of the $y$ 's do not necessarily have exponents at all points of $(J)$. They may for instance vanish an infinite number of times in $(J)$. 


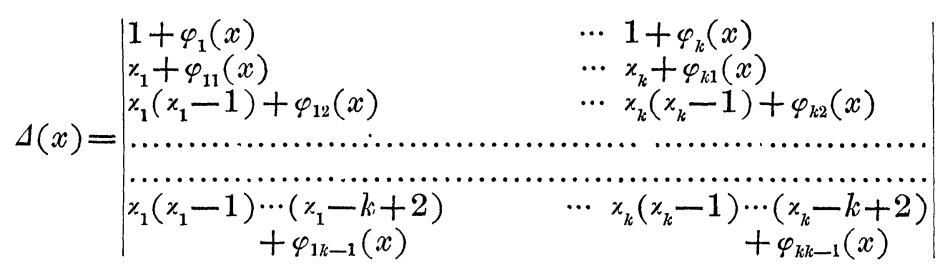

Throughout $(J) J(x)$ is continuous and, since all the $\varphi$ 's vanish at $c, \Delta(c)$ is equal to the product of the differences of the $x$ 's. No two of the $x$ 's being equal, $\Delta(c) \neq 0$.

Thus we see from (12) that $W\left(\eta_{1}, \cdots, \eta_{k}\right)$, and therefore also $W\left(y_{1}, \cdots, y_{k}\right)$, has at $c$ the exponent

$$
x=x_{1}+x_{2}+\cdots+x_{k}-\frac{k(k-1)}{2} .
$$

The least possible value of $x$ is obtained by giving to $x_{1}$, $x_{2}, \cdots, x_{k}$ the values $0,1, \cdots, k-1$; and the greatest possible value by giving them the values $n-k, n-k+1, \cdots, n-1$. Therefore

$$
0 \leqq x \leqq k(n-k) \text {. }
$$

Combining the theorem just proved with II., we see that

X. $W\left(y_{1}, \cdots, y_{k}\right)$ cannot vanish an infinite number of times in $(J) * *$ fore

When $W\left(y_{1}(c), y_{2}(c), \cdots, y_{k}(c)\right)+0$ we have $x=0$. There-

XI. Except at the points where $W\left(y_{1}, \cdots, y_{k}\right)=0$, the family (6) has the exponents $0,1, \cdots, k-1$.

Another consequence of (13), a direct proof of which is also very simple, is that, when $k=n, W\left(y_{1}, \cdots, y_{n}\right)$ vanishes nowhere in $(J) \cdot \dagger$

Finally we introduce the conception of sub-families as follows :

Let $\eta_{1}, \eta_{2}, \cdots, \eta_{m}(m<k)$ be linearly independent functions of the family $(6)$. With these functions as a basis we construct a family (7), which since it is wholly contained in (6) we call a sub-family of (6). These sub-families all satisfy the conditions stated at the beginning of this section,

* A special case of this is the theorem that $W\left(y_{1}, \cdots, y_{k}\right)$ is not identically zero. It should be noticed that we thus get a new proof of this theorem since we have made no use of it up to this point.

† Conversely it can readily be proved that if $y_{1}, \cdots, y_{n}$ are any functions which are continuous and have continuous derivatives of the first $n-1$ orders throughout $(J)$ and whose Wronskian does not vanish in $(J)$, then they form a basis of a linear family, none of whose members, except zero, vanishes together with its first $n-1$ derivatives at a point of $(. J)$. 
and hence theorems IX., X., XI., apply to them. In particular we see that the Wronskian of any set of linearly independent functions of the family has an exponent at every point of $(J)$.

§. The Adjoint Family.

We will begin here by assuming merely that $y_{1}, \cdots, y_{k}$ are continuous and linearly independent throughout $(J)$ and have throughout this interval finite derivatives of the first $k-2$ orders.

Let us consider the Wronskians

$$
W_{i}=W\left(y_{1}, \cdots, y_{i-1}, y_{i+1}, \cdots, y_{k}\right) \quad(i=1,2, \cdots, k)
$$

Let $\theta_{1}, \theta_{2}, \cdots, \theta_{k-1}$, be any $k-1$ functions of the family (6), say

$$
\theta_{i}=a_{i 1} y_{1}+a_{i 2} y_{2}+\cdots+a_{i k} y_{k}(i=1,2, \cdots, k-1) .
$$

It is at once seen that

$$
W\left(\theta_{1}, \cdots, \theta_{k-1}\right)=c_{1} W_{1}+c_{2} W_{2}+\cdots+c_{k} W_{k},
$$

where

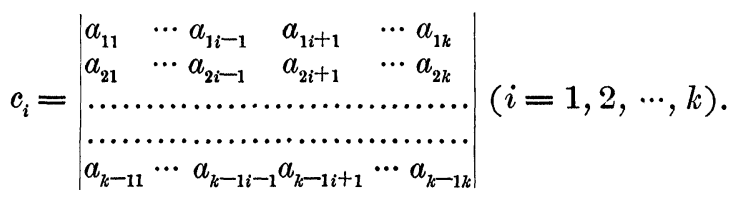

Conversely if the constants $c$ are given, the $a$ 's can be so determined that equations (17), and therefore also equations (16), hold.* That is,

XII. The Wronskians of the functions of the family (6) taken $k-1$ at a time themselves form a linear family.

Moreover the proof just given shows that either the functions $W_{1}, \cdots, W_{k}$ or, if they are linearly dependent, some functions taken from among them form a basis of this new family. In this last case we should have a relation of the form

$$
c_{1} W_{1}+\cdots+c_{k} W_{k}=0,
$$

where the $c$ 's are not all zero. The $a$ 's being then computed

* If all the $c$ 's are zero this is obvious If not, suppose $c_{1} \neq 0$. We have then merely to choose all the $a^{\prime}$ s except $a_{11}, a_{21}, \cdots, a_{k-11}$ so that when $i=1$ (17) is satisfied, and then to determine the remaining $a$ 's by means of the equations

$$
a_{i 1} c_{1}-a_{i 2}(2)+\cdots+(-1)^{k-1} a_{i k} r_{k}=0 \quad(i=1,2, \cdots, k-i) .
$$


as above from (17), the $\theta$ 's would be linearly independent, but $W\left(\theta_{1}, \cdots, \theta_{k-1}\right)=0$. Thus we have the theorem

XIII. The functions $W_{1}, \cdots, W_{k}$ are linearly independent unless there exist $k-1$ linearly independent functions in the family (6) whose Wronskian vanishes identically.*

Let us now impose on the functions $y_{1}, \cdots, y_{k}$ the further restrictions which are stated at the beginning of $\S 3$. The $k$ functions

$$
z_{i}=(-1)^{k+i} \frac{W_{i}}{W\left(y_{1}, \cdots, y_{k}\right)} \quad(i=1,2, \cdots, k)
$$

we call $\dagger$ the functions adjoint to $y_{1}, \cdots, y_{k}$. We then have the theorem

XIV. The functions formed by dividing the Wronskians of the functions of (6) taken $k-1$ at a time by the Wronskian of a basis of (6) themselves form a linear family-the family adjoint to (6). The functions adjoint to any basis of (6) form a basis of this adjoint family.

To this may be added, if we refer to (13),

$\mathrm{XV}$. If $\eta_{1}, \cdots, \eta_{k}$ form a principal basis of (6) at the point $c$, then the adjoint functions $\zeta_{1}, \cdot \cdot, \zeta_{k}$ form a principal basis of the adjoint family at $c$. If the exponent of $\eta_{i}$ at $c$ is $x_{i}$, the exponent of $\zeta_{i}$ will be $k-1-x_{i}$.

\section{\$5. Applications to Differential Equations.}

We will now consider the differential equation

$$
P(y) \equiv \frac{d^{n} y}{d x^{n}}+p_{1} \frac{d^{n-1} y}{d x^{n-1}}+\cdots+p_{n} y=0,
$$

where $p_{1}, \cdots, p_{n}$ are throughout $(J)$ continuous functions of the real variables $x$. Theorems IX. and $\mathrm{X}$. give us at once

XVI. If $y_{1}, \cdots, y_{k}$ are linearly independent solutions of (19), and if $c$ is any point of $(J)$ then $W\left(y_{1}, \cdots, y_{k}\right)$ has an integral exponent $x$ at $c$, where

$$
0 \leqq x \leqq k(n-k) \text {. }
$$

XVII. The Wronskian of $k$ linearly independent solutions of (19) cannot vanish an infinite number of times in $(J)$.

Let us now look at the question of determining the multipliers of the equation (19), that is functions $z$ such that

\footnotetext{
* Sufficient conditions that this should not occur will be found in my paper in the Transactions, vol.2, p. 139.

$\dagger$ Frobenius introduced this term in Crelle, vol. 77, p. 250.
} 


$$
z P(y)=\frac{d}{d x}\left[q_{1} \frac{d^{n-1} y}{d x^{n-1}}+q_{2} \frac{d^{n-2} y}{d x^{n-2}}+\cdots+q_{n} y\right]
$$

It is clear that we must here have

$$
z=q_{1} \text {. }
$$

Equation (20) then becomes equivalent to the following set of equations:

$$
\begin{aligned}
& q_{1}^{\prime}=p_{1} q_{1}-q_{2}, \quad q_{2}^{\prime}=p_{2} q_{1}-q_{3}, \\
& q_{n-1}^{\prime}=p_{n-1} q_{1}-q_{n}, \quad q_{n}^{\prime}=p_{n} q_{1} \text {. }
\end{aligned}
$$

These form a set of simultaneous linear homogeneous differential equations of the first order for determining the $q$ 's. It is customary to eliminate $q_{2}, \cdots, q_{n}$ between equations (21) and thus get a homogeneous linear differential equation of the $n$th order-the equation adjoint to (19)-for $q_{1}$ (or $z$ ).* This method, however, is not open to us unless we are willing to assume that the coefficients, $p_{1}, p_{2}, \cdots, p_{n-1}$ have derivatives of orders $n-1, n-2, \cdots, 1$ respectively.

From equations (21) we infer directly that the multipliers of (19) form a linear family whose bases consist of $n$ functions each. This family is in fact adjoint to the family of solutions of (19). This last fact is most readily proved by establishing by the method of Frobenius $\dagger$ the formula

* We obtain of course in the same way equations for the other $q$ 's. The equation satisfied by $q_{i}$ has been termed the $(n-i+1)$ th adjoint equation, so that what is ordinarily called simply the adjoint equation would be the $n$th adjoint equation. Cf. Cels, Ann. de l'Ec. norm. sup., $3 \mathrm{~d}$ ser., vol. 8 (1891), p. 341, and some more recent papers in Crelle.

† Cf. Crelle, vol. 77 (1874) p. 248. The method consists of deducing (22) from a special case of the important general formula

where

$$
\begin{gathered}
W\left(y_{1}, \cdots, y_{k}\right)\left[W\left(y_{1}, \cdots, y_{m}\right)\right]^{k-m-1}=W\left(w_{m 1}, w_{m 2}, \cdots, w_{m k-m}\right) \\
(1 \leqq m \leqq k-1)
\end{gathered}
$$

$$
w_{m i}=W\left(y_{1}, \cdots, y_{m}, y_{m+i}\right) \quad(i=1,2, \cdots, k-m) .
$$

Frobenius's method of proving the formula $(F)$ consists in first establishing the formula directly when $m=1$, and then using the method of mathematical induction. Each of these two steps requires a slight addition to make it rigorous when we are dealing with non-analytic functions of a real variable. Thus what we establish by the general step in the mathematical induction is

$$
\begin{gathered}
W\left(y_{1}, \cdots, y_{k}\right)\left[W\left(y_{1}, \cdots, y_{m}\right)\right]^{k-m-1}\left[W\left(y_{1}, \cdots, y_{m-1}\right)\right]^{k-m} \\
=W\left(w_{m 1}, w_{m 2}, \cdots, w_{m k-m}\right)\left[W\left(y_{1}, \cdots, y_{m-1}\right)\right]^{k-m}
\end{gathered}
$$




$$
z_{i} P(y)=\frac{d}{d x}\left[(-1)^{i-1} \frac{W\left(y \cdot y_{1}, \cdots, y_{i-1} \cdot y_{i+1}, \cdots, y_{n}\right)}{W\left(y_{1}, \cdots, y_{n}\right)}\right]
$$

where $y_{1}, \cdots, y_{n}$ are any set of $n$ linearly independent solutions of (19) and $z_{1}, \cdots, z_{n}$ are the functions adjoint to them. Thus we have proved the theorem

XVIII. A necessary and sufficient condition that $z$ be a multiplier of (19) is that it be a member of the linear family adjoint to the family which consists of the solutions of (19).

GRUND IM HARZ,

July 20, 1901 .

\section{THE CONFIGURATIONS OF THE 27 LINES ON A CUBIC SURFACE AND THE 28 BITAN- GENTS TO A QUARTIC CURVE.}

BY PROFESSOR L. E. DICKSON.

(Read before the American Mathematical Society, August 20, 1901.)

\section{Introduction.}

AfTER determining * four systems of simple groups in an arbitrary domain of rationality which include the four sys. tems of simple continuous groups of Lie, the writer was led to consider the analogous problem for the five isolated sim. ple continuous groups of $14,52,78,133$, and 248 parameters. The groups of 78 and 133 parameters are related to certain interesting forms of the third and fourth degrees respectively. $\dagger$ They suggested the forms $C(\S 1)$ and $Q(\S 3)$.

It is shown in $\$ 1$ that the cubic form $C$ defines the configuration of the 27 straight lines on a cubic surface in or-

the functions $y_{1}, \cdots, y_{k}$ being supposed to be any functions of $x$ which throughout $(J)$ have continuous derivatives of the first $k-1$ orders. This establishes the truth of $(F)$ at all points of $(J)$ except where

$$
W\left(y_{1}, \cdots, y_{m-1}\right)=0 \text {. }
$$

If $c$ is a point where this last equality holds two cases are possible: $1^{\circ}$ there may be points in every neighborhood of $c$ where the equality does not hold and where therefore $\left(F^{*}\right)$ holds. In this case, on account of the continuity of both sides of $(F)$, this formula holds also at $c 2^{\circ}$, $W\left(y_{1}, \cdots, y_{m-1}\right)$ may vanish identically throughout the neighborhood of $c$. In this case $(F)$ also holds at $c$ since all the Wronskians which occur in it vanish at $c$; ef. Transartions, vol. 2 , p. 148.

* Abstract presented to the Society, Aug. 20, 1901, to appear in extenso in the Transactions. A note on the subject appeared in Comptes icndus, CXXXII. (1901), pp. 1547-8.

† Cartan, Thèses, Paris, 1894. 